\title{
Estimation of material degradation of VVER-1000 baffle
}

\author{
Davit Harutyunyan ${ }^{1,2, *}$, Michal Košt'ál ${ }^{2}$, Stanislav Vandlík ${ }^{2}$, Anna Hojná2, Martin Schulc ${ }^{2}$, and Stanislav Flibor ${ }^{2}$ \\ ${ }^{1}$ Department of Nuclear Reactors, FNSFE, CTU Prague, V Holešovičkách 2, Prague 8, Czech Republic \\ ${ }^{2}$ Research Center Rez Ltd., 25068 Husinec-Rez 130, Czech Republic
}

\begin{abstract}
The planned lifetime of the first commercial VVER-1000 units were designed for 30 to 35 years. Most of the early VVER plants are now reaching and/or passing the 35-year mark. Service life extension for another 10 to 30 years is now under investigation. Life extension requires the evaluation of pressure vessel internals degradation under long-term irradiation. One of the possible limiting factors for the service life of VVERs is a void swelling of the Russian type titanium stabilized stainless 08Ch18N10T steel used to construct the baffle surrounding the core. This article aims to show first steps towards deeper analysis of the baffle degradation process and to demonstrate the possibilities of precise calculation and measurements on the VVER-1000 mock-up in LR-0 reactor.
\end{abstract}

\section{Introduction}

At present, more knowledge of void swelling formation and development in the thermal reactor conditions is needed. Reliable data of voids swelling have been obtained in conditions of different neutron spectrum - in fast reactors or accelerators [1], [2]. Data shows $20 \%$ and higher void swelling presence in the steels results in a drastic and total loss of strength and plasticity, but the findings were obtained for the steels irradiated up to $72 \mathrm{dpa}$ at fast neutron spectrum (BOR-60 reactor) at about $400-550^{\circ} \mathrm{C}$.

Certainly, the impact of voids swelling on material behavior, especially on mechanical strength or fracture resistance is very devastating, but it is a question if these warnings are applicable to Light Water Reactors LWRs, because total void swelling observed to date in Flux Thimble Tube of Pressure Water Reactors PWRs, up to 80 displacement per atom dpa, has been relatively low, under $1 \%$ [3]. One dpa means that every atom has been displaced once on average. It is material dependent measure of an exposure.

\section{Void swelling in VVER -1000 baffle}

The degradation effect from gamma rays in the case of VVER-1000 reactor pressure vessel (RPV) does not exceed $4 \%$ of total dpa. In case of baffle the source of material degradation is mainly by high energy gamma origin by neutron capture.

Our calculations for VVER-1000 mock-up assembled at LR-0 zero power experimental reactor (Research Centre Rez) show that neutron share on radiation heating in thicker parts of baffle is less than 3 $\%$. Deposited energy from radiation heating is transferred to the steel lattice causing atomic displacements - radiation damage. The damage causes changes of material behavior including some void swelling which results an increase in the external dimension of solid materials after irradiation.

Void swelling of the reactor internal parts is closely related to the temperature at the site. Increasing temperature causes higher probability of gas formation and trapping it in the barrel material. This temperature may be significantly higher than the temperature of the coolant in internal parts of reactor because there is significant heating due to gamma radiation. Gamma caused heating got origin by fission, decay of fission products in fuel or neutron interactions. Mainly in thicker parts of the baffle, an important role is played by the gamma originated by thermal capture on steel (7.63MeV and 7.65 MeV) or on ${ }_{1}^{1} \mathrm{H}(2.23 \mathrm{MeV})$.

Impact of gamma irradiation from capture on ${ }_{1}^{1} \mathrm{H}$ is notable in vicinity of cooling channels (1-13 on the Fig.1). Since, these reactions are caused mainly by thermal neutrons, theirs correct description is fundamental for accurate gamma field distribution description. Temperature shift in baffle material is caused mainly by gamma heating in contrast to the surrounding water. Eq. 1 estimates void swelling originated by radiation impact [4]:

$$
S(k, D, T)=R(k) \varphi\left(D-D_{0}(k, T)\right) \exp \left\{-\frac{\left(T-T_{\max }(k)\right)^{2}}{2 \sigma_{T}^{2}(k)}\right\}(1)
$$

where $S(k, D, T)$ is the swelling $(\%), D$ is the damage dose (dpa), $T$ is the irradiation temperature $\left({ }^{\circ} \mathrm{C}\right), k$ is the dose rate $(\mathrm{dpa} / \mathrm{s}), \varphi(x)=x \cdot \theta(x), \theta(x)$ is Heaviside unit step function, $\theta(x)=1, x>0$ or $0, x \leq 0$,

\footnotetext{
* Corresponding author: davit.harutyunyan@cvrez.cz
} 
$R(k)=r_{0}-r_{k} \ln \mathrm{k}$ is the swelling rate at steady state $(\% / \mathrm{dpa}), D_{0}(k, T)=d_{0}-d_{T} T+d k \ln \mathrm{k}$ is the incubation period (dpa), $T_{\max }(k)=T_{0}+T_{k} \ln k$ is the peak swelling temperature $\left({ }^{\circ} \mathrm{C}\right), \sigma_{t}(k)=\sigma_{0}-\sigma_{k} \ln k$ is the temperature dispersion $\left({ }^{\circ} \mathrm{C}\right)$.

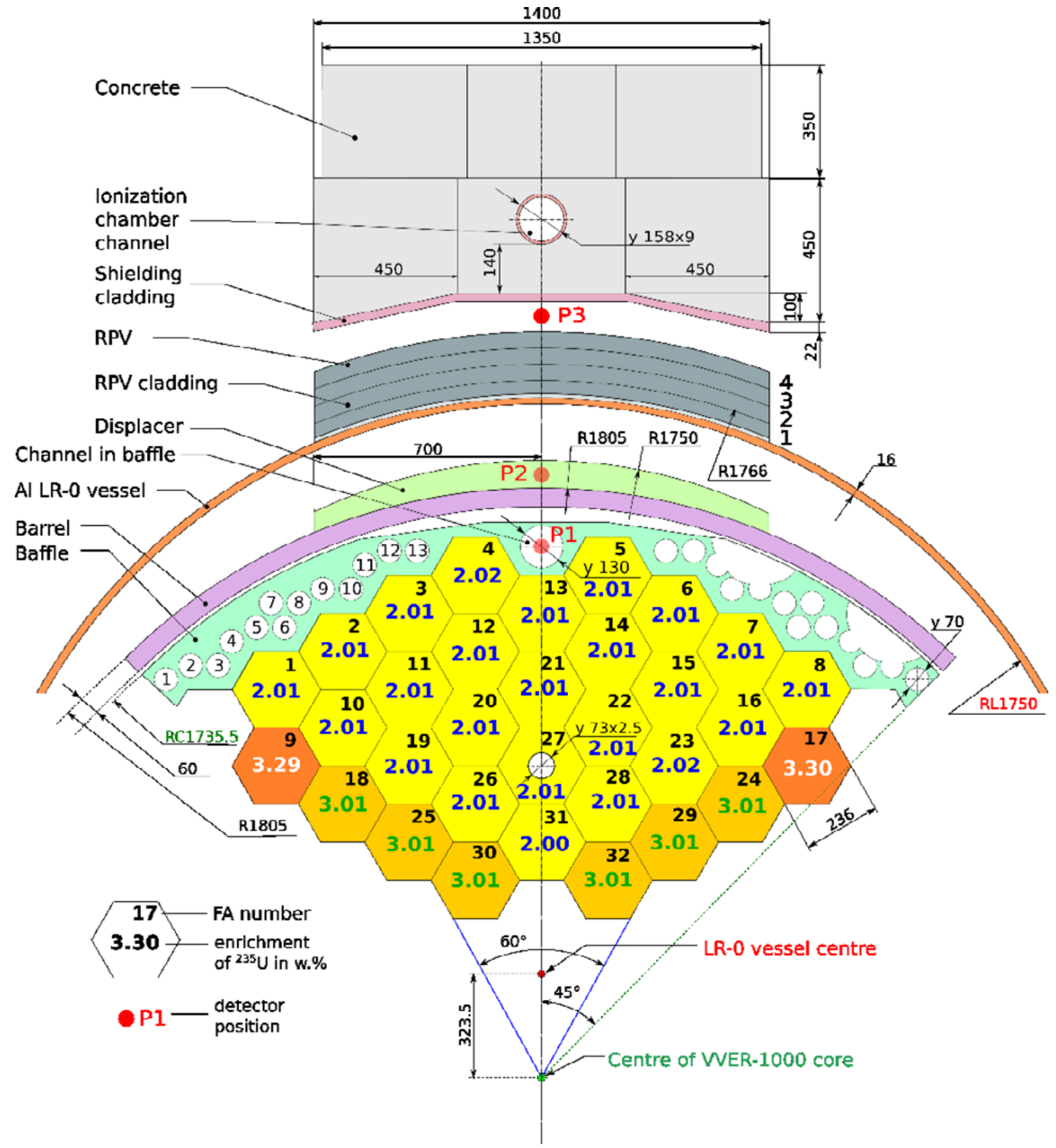

Fig. 1 Section of the VVER-1000 mock-up in LR-0 reactor in the $x-y$ plane (Dimensions in mm).

Empirical equation with dependence on dpa rate was employed in computations. The creation of Frenkel pairs - dpa according to widely used NRT theory [5] is given by the Kinchin-Pease see formula 2 .

$$
D P A=k \frac{E_{a}}{2 E_{d}}
$$

where $E_{a}$ stands for available energy, $E_{d}$ for the Lindhard cut-off energy required for displacing one atom from its normal site and $\mathrm{k}$ for displacement efficiency. However, this formula does not account for any thermally activated recombination of damage, nor the well-known fact that in metals the damage production is for high energies only approximately $20 \%$ of the Kinchin-Pease prediction [6]. Formula also does not take into account ion channelling, which may in crystalline or polycrystalline materials reduce the nuclear deposited energy and thus the damage production dramatically for some ion-target combinations. This suggests that calculated value is upper limit of dpa estimate. Incident 
particles of different types and energies produce different types of primary damage (neutrons, heavy charged particles, electrons, photons), they produce differences in secondary damage accumulation. To estimate radiation damage we need to know both energy spectrum and absolute flux level. Fission fragments have limited range, primarily impacts the fuel. Most important source of dislocations in the baffle is caused by fast neutrons.

\section{Experimental and calculation methods}

Displacement per atom and gamma heating calculations were done with MCNP6 model of LR-0 VVER-1000 mock-up. This model is validated with experimental data gathered on VVER-1000 mock-up measurements [7] [8] [9]. The MCNP calculations provide data which are impossible to measure, such as deposited energy of particles. Completing this data with thermo-hydraulic computation, it is possible to obtain all values in Eq. 1 for the void swelling determination. To obtain required data for void swelling determination approximately $27 \times 23 \mathrm{~cm}$ part of reactor baffle was chosen, see Fig. 2 .
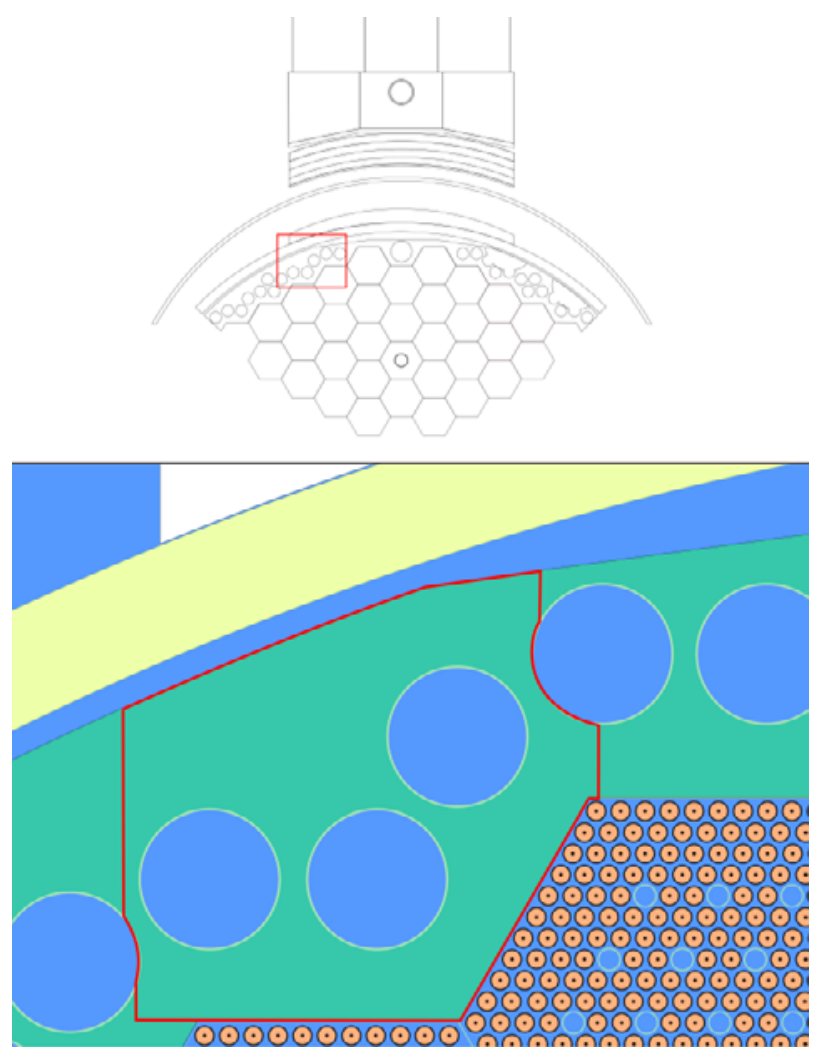

Fig. 2 Selected part of baffle.

\subsection{Experimental methods}

The mock-up core consists of 32 dismountable assemblies with enrichment $2 \%, 3 \%$ and $3.3 \%$ of 235U. Demineralized water with dissolved boric acid with $4.6 \pm 0.1 \mathrm{~g} / \mathrm{l}$ is used as a moderator. The fuel assemblies are of shortened VVER type, (sintered UO2 pellets, outer diameter $7.53 \mathrm{~mm}$, central inner hole 1.4 $\mathrm{mm}$, active length $125 \mathrm{~cm}$, total length $135.7 \mathrm{~cm}, \mathrm{Zr}$ cladding tube with outer diameter $9.15 \mathrm{~mm}$, wall thickness $0.72 \mathrm{~mm}$ ). Mock-up includes radially full scale VVER-1000 barrel, baffle simulators and concrete shielding. For computational models validation the neutron spectra measurements were used in points P2 and P3 (see Fig.1). Neutron spectra below $1 \mathrm{MeV}$ were measured using proton recoiled method with a hydrogen chamber, which was operated at $400 \mathrm{kPa}$ over an energy range from $0.111 \mathrm{MeV}$ to $0.368 \mathrm{MeV}$ and at $1000 \mathrm{kPa}$ in the higher range. For energies from $1 \mathrm{MeV}$ to $10 \mathrm{MeV}$ a stilbene scintillator was used with neutron and gamma pulse shape discrimination. Details of this measurement could be found in [10]

\subsection{Calculation methods}

Gamma heating and dpa were calculated via MCNP6 model of VVER-1000 mock-up, using mesh tally for the chosen part. TORT calculations were used for verification of MCNP results.

\subsubsection{MCNP model}

MCNP computation model is fixed source: where the neutron emission from fuel is defined directly with external sources and fission is dealt as absorption, this is mainly done for the computation time saving. The model geometry is maximally realistic and detailed including stainless steel components of the reactor core, baffle and barrel models up to the concrete biological shielding. This model is detailed described in [7]. The fixed source model uses directly defined neutron or gamma emission from the fuel. Neutron emission density was defined by means of fission density obtained in critical calculation in MCNP. The total gammas in selected part of baffle Fig. 2 are coming from the primary gamma from the fission and secondary. The primary gamma is absent in the case of fixed neutron source calculation. They are computed with additional calculation with gamma source in fuel. To calculate the available energy $E_{a}$ from the Eq. 2, flux mesh tally (flux averaged over the grid cell) has to be modified by the damage energy production cross section library IRDF-2002 [12]. The Lindhard cut-off energy and displacement efficiency are chosen according to ref. [11]: $E_{d}=40 \mathrm{eV}$ and $k=0.8$ for major steel components. ENDF VII.1 [13] nuclear data library were used for the calculation model compilation.

\subsubsection{TORT model}

Used TORT model is composed of $1 / 6^{\text {th }}$ energetic VVER-1000 reactor core, i.e. 28 fuel assembles of VVER-1000 reactor core and all components from baffle to concrete shielding are included. The abovementioned TORT 3D model is currently used for neutron and gamma flux calculations for nuclear power plant (NPP). The cross section library used for presented calculations is BUGLE-96 (RSICC, 1999) 47-neutrongroup/20-gamma-ray-group collapsed library based on ENDF/B-VI. This model is described in detail in [7]. 


\section{Results}

Presented results show the dpa and gamma heating maps in the chosen region of baffle. Calculated results with MCNP for mock-up were scaled to the VVER-1000 nominal power of $3000 \mathrm{MW}$ thermal power of energetic nuclear power plant. As it is obvious from the Fig. 3 and 4 the distribution of dpa and gamma heating is very similar in both calculations. In both cases the distribution is taken from the middle of core height. TORT calculation is done for

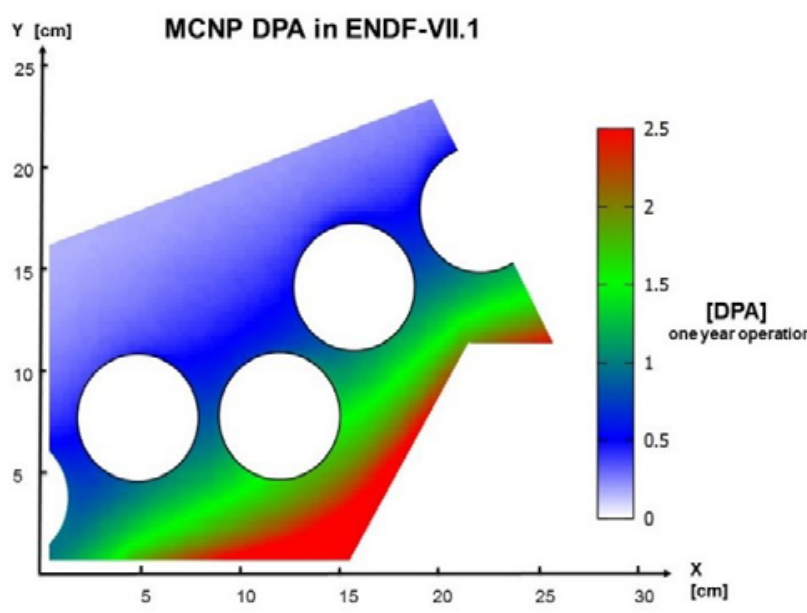

Fig. 3 Relative distribution of dpa in the selected part of baffle

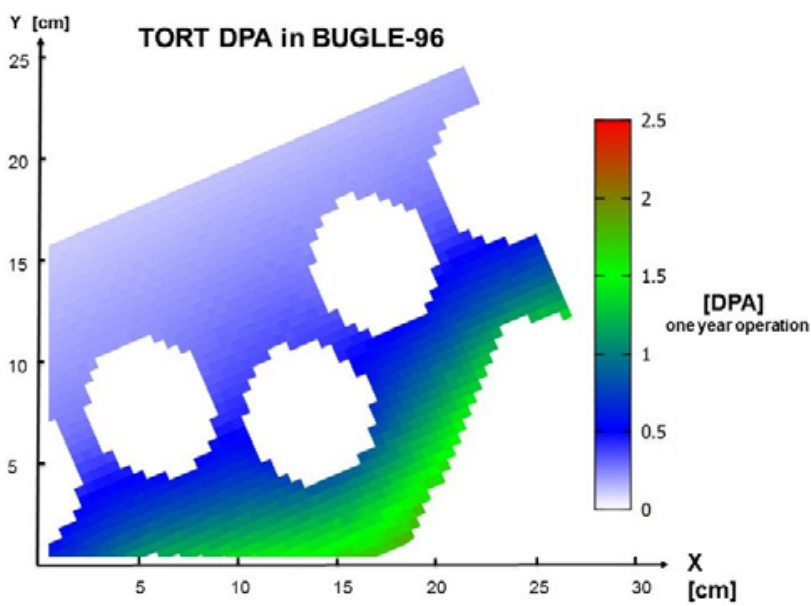

Fig. 4 Relative distribution of dpa in the selected part of baffle

the energetic reactor and there are several differences compared with MCNP in initial data, mainly composition of fuel (burn up included in case of TORT) fuel length: $125 \mathrm{~cm}$ in case of MCNP model and $350 \mathrm{~cm}$ in case of TORT model, length of fuel cycle and others described in [7].

Gamma heating map presented below Fig. 5 and 6 were obtained using same technique as in the case of dpa. In both cases the distribution is similar and the differences in absolute values are mainly because of above mentioned reasons.

\section{Uncertainties and validation}

Calculation uncertainty covers statistical uncertainty of calculation less than 1\% MCNP or convergence criterion of 0.001 in case of TORT. The statistical uncertainties can be neglected, as they are negligible with regard to parameters uncertainties. For parameters uncertainties estimation the sensitive analysis were performed.

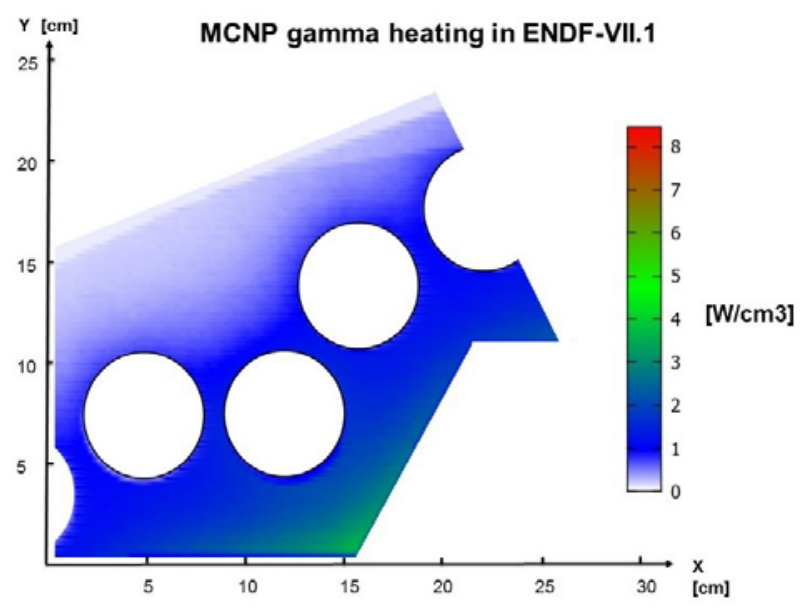

Fig. 5 Relative gamma heating map in the selected part of baffle

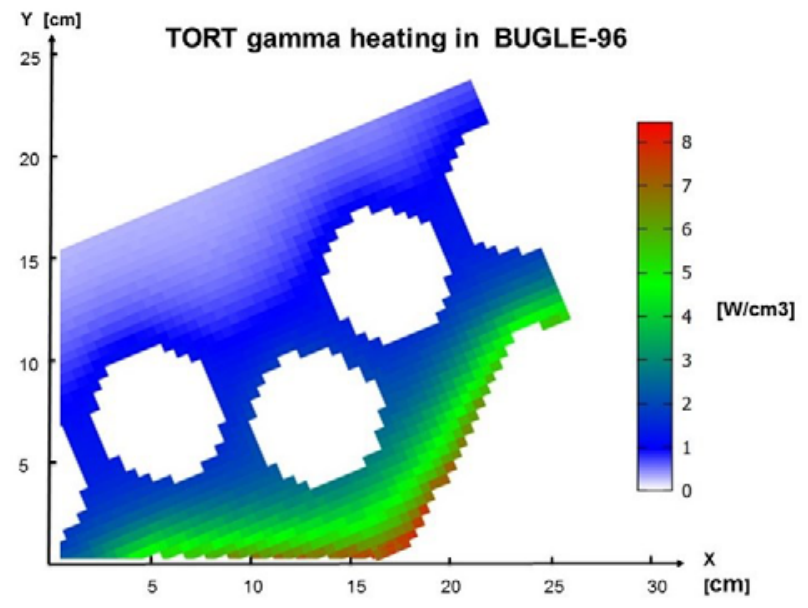

Fig. 6 Relative gamma heating map in the selected part of baffle

The main sources of calculation uncertainties are baffle steel density, boric acid concentration, fuel enrichment and gap between fuel pins and the baffle.

For dpa the main contributions to uncertainty are baffle steel density up to $1.4 \%$, boric acid concentration up to $0.12 \%$, fuel enrichment up to $0.25 \%$ and gap distance up to $0.8 \%$ giving total uncertainty in dpa $1.6 \%$.

For gamma heating the corresponding contributions to uncertainty are baffle steel density up to $2.7 \%$, boric acid concentration up to $0.15 \%$, fuel enrichment up to $1.4 \%$ and gap distance up to $3.0 \%$ giving total uncertainty in gamma heating values $4.3 \%$. The total standard uncertainty was determined by summing the values of square standard uncertainty for all of the parameters and taking the square root to convert the variance to a standard uncertainty. 
Due to considerable differences in MCNP and TORT geometries the relative neutron fluxes are calculated in 3 specified points P1-P3, which are shown on the Fig. 1 and compared with measured spectra in the point P2 and P3. Fig. 7 shows relative neutron spectra for MCNP, TORT and experiment. Considering good agreement between the models and measurement the results of dpa and gamma heating calculations seem reasonable. The measurement uncertainties can be found in [10].

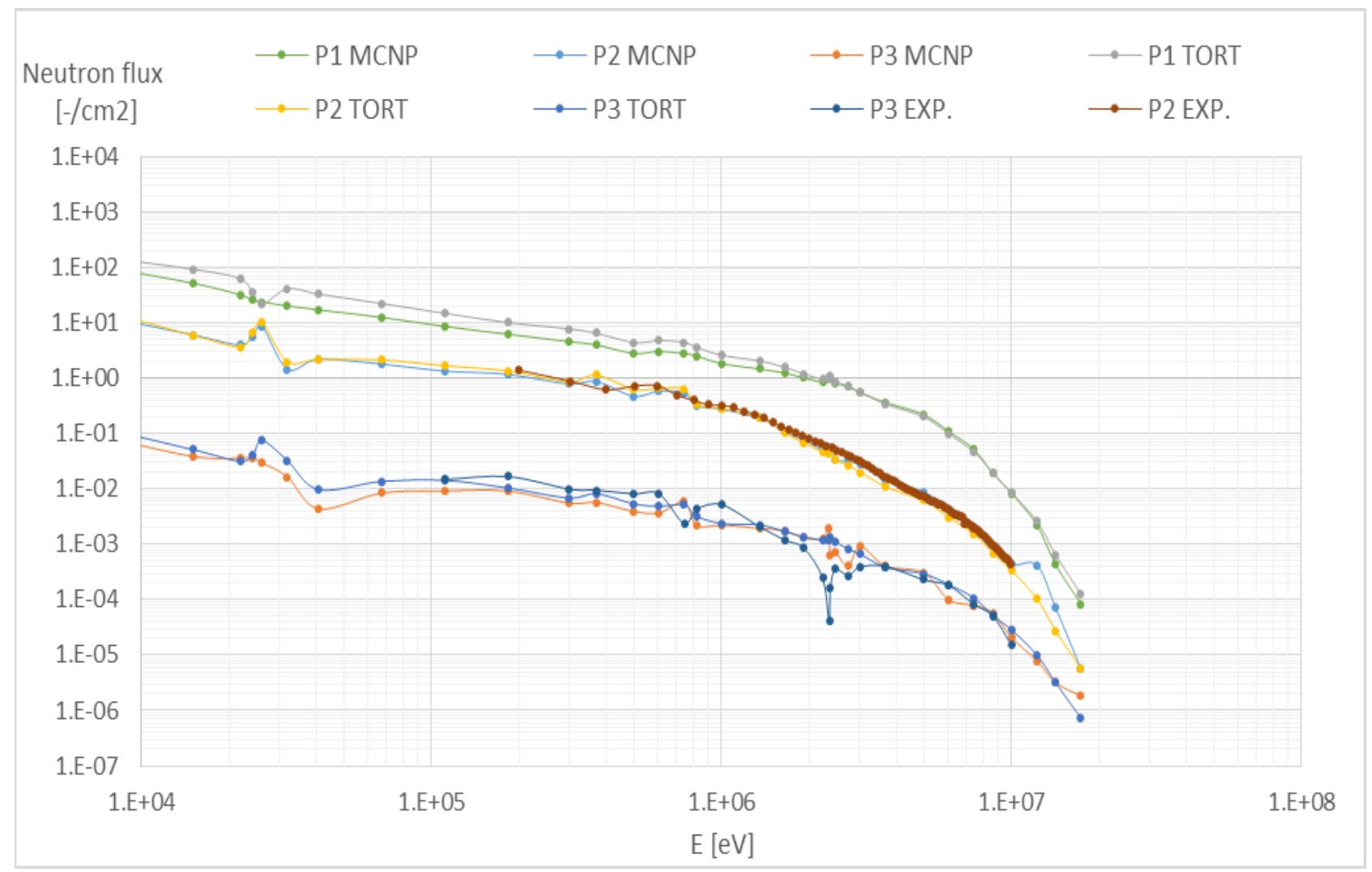

Fig. 7 Relative neutron spectra in position 1,2 and 3

\section{Conclusions}

MCNP results for dpa and gamma heating in selected part of baffle are in an agreement with TORT results. Both distributions are comparable. Maximum dpa integrated over a year was estimated using MCNP as 2.5 and by TORT as 1.5 dpa. Maximum gamma heating in MCNP calculation is $4 \mathrm{~W} / \mathrm{cm} 3$ compared with $8 \mathrm{~W} / \mathrm{cm} 3$ in case of TORT. Used methods for absolute dpa and gamma heating estimation via MCNP calculations seems to be applicable for the energetic VVER-1000 reactor. The reason for a variance in the absolute values are due to the slight differences in nuclear parameters, geometry definition of computational models and by scaling process in MCNP. Dpa and gamma heating are the input data for void swelling estimation. In accordance with our experience we regard stochastic codes (MCNP) to be more reliable and with smaller margin of error, than codes using deterministic method.

\section{Acknowledgement}

The presented work was financially supported by the grant for Project SGS16/174/OHK4/2T/ 4, FNSPE of the
CTU, department of Nuclear Reactors and the Ministry of Education, Youth and Sport Czech Republic Project LQ1603 (Research for SUSEN).

\section{References}

1. V.S. Neustroev, F.A. Garner / Journal of Nuclear Materials 386-388 (2009) 157-160.

2. B.Z. Margolin, I.P. Kursevich, A.A. Sorokin, V.S. Neustrojev: "The Relationship of Radiation Embrittlement and Swelling for Austenitic Steels for WWER Internals", Proceedings of the ASME 2009, Pressure Vessels and Piping conference, Prague, 2009.

3. J. L. Nelson et al., Proceedings of the 16th International Symposium on Environmental Degradation of Materials in Nuclear Power Systems - Water Reactors, Asheville, USA, 2013.

4. A.S. Kalchenko, V.V. Bryk, N.P. Lazarev, V.N. Voyevodin, F.A. Garner, Prediction of void swelling in the baffle ring of WWER-1000 reactors for service life of 30-60 years, Journal of Nuclear 
Materials, Volume 437, Issues 1-3, June 2013, Pages 415-423, ISSN 0022-3115.

5 Norgett, M. J., Robinson, M. T. and Torrens, I. M. A proposed method of calculating $\mathrm{v}$ displacement dose rates. Nucl. Eng. Des. 33, 50-54 (1975).

6 R. S. Averback and T. Diaz de la Rubia, Displacement damage in irradiated metals and semiconductors, in Solid State Physics, edited by H. Ehrenfest and F. Spaepen, volume 51, pages 281-402, Academic Press, New York, 1998.

7 D. Harutyunyan, S. Vandlik, M. Schulc, M. Ruscak, E. Novak, Applicability of LR-0 mock-up results to VVER-1000 reactor pressure vessel issues, Annals of Nuclear Energy, Volume 98, December 2016, Pages 157-165, ISSN 0306-4549.

8 M. Košt'ál, V. Rypar, M. Švadlenková, The pin power distribution in the VVER-1000 mock-up on the LR-0 research reactor, Nucl. Eng. Des., 242 (2012), pp. 201-214 ISSN 0029-5493

9 M. Košt’ál, M. Švadlenková, J. Milčák, Absolute determination of power density in the VVER-1000 mock-up on the LR-0 research reactor, Appl. Radiat. Isot., 78 (2013), pp. 38-45 ISSN 0969-8043

10 M. Košt'ál, F. Cvachovec, V. Rypar, V. Juříček, Calculation and measurement of neutron flux in the VVER-1000 mock-up on the LR-0 research reactor, Annals of Nuclear Energy, Volume 40, Issue 1, February 2012, Pages 25-34, ISSN 0306-4549

11 Greenwood, L. R. and Smither, R. K. SPECTER; neutron damage calculations for materials irradiations. ANL/FPP/TM-197. Argonne National Laboratory (1985).

12 IAEA/NDS IRDF-2002 Damage Cross Section File 2002, https://wwwnds.iaea.org/irdf2002/data/irdf2002_damage.dat

13 M.B. Chadwick, et al. s.l.: ENDF/B-VII.1 Nuclear data for science and technology: cross sections, covariances, fission product yields and decay data Nucl. Data Sheets, 112 (2011), pp. 2887-2996 